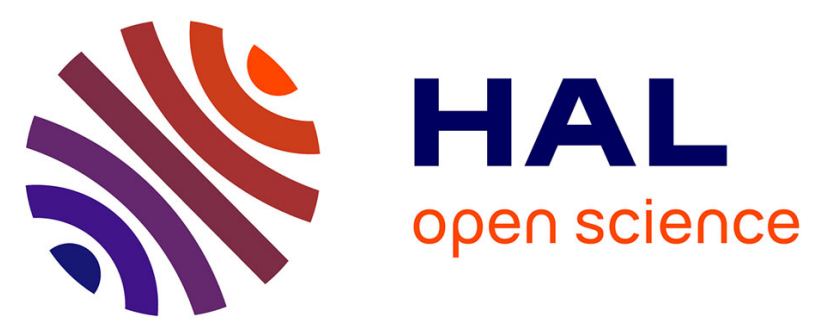

\title{
An Analytical Model for Probing Ion Dynamics in Clays with Broadband Dielectric Spectroscopy
}

\author{
Benjamin Rotenberg, Anthony Cadene, Jean-François Dufrêche, Serge
}

Durand-Vidal, Jean-Claude Badot, Pierre Turq

\section{- To cite this version:}

Benjamin Rotenberg, Anthony Cadene, Jean-François Dufrêche, Serge Durand-Vidal, Jean-Claude Badot, et al.. An Analytical Model for Probing Ion Dynamics in Clays with Broadband Dielectric Spectroscopy. Journal of Physical Chemistry B, 2005, 109, pp.15548-15557. 10.1021/jp051586k . hal-00166966

\section{HAL Id: hal-00166966 https://hal.science/hal-00166966}

Submitted on 9 Nov 2018

HAL is a multi-disciplinary open access archive for the deposit and dissemination of scientific research documents, whether they are published or not. The documents may come from teaching and research institutions in France or abroad, or from public or private research centers.
L'archive ouverte pluridisciplinaire HAL, est destinée au dépôt et à la diffusion de documents scientifiques de niveau recherche, publiés ou non, émanant des établissements d'enseignement et de recherche français ou étrangers, des laboratoires publics ou privés. 


\title{
An analytical model for probing ion dynamics in clays with Broadband Dielectric Spectroscopy
}

\author{
B. Rotenberg ${ }^{1, *}$, A. Cadéne ${ }^{1}$, J.-F. Dufrêche ${ }^{1}$, \\ S. Durand-Vidal ${ }^{1}$, J.-C. Badot ${ }^{2}$ and P. Turq ${ }^{1}$
}

June 20, 2005

${ }^{1}$ Laboratoire Liquides Ioniques et Interfaces Chargées (UMR CNRS 7612), Université P. et M. Curie, 4 place Jussieu, 75252 Paris Cedex 05, France

${ }^{2}$ Laboratoire de Chimie Appliquée de l'Etat Solide (UMR CNRS 7574), ENSCP, 11 rue P. et M. Curie, 75231 Paris Cedex 05, France

\begin{abstract}
A simple two-state model is proposed to explicitly derive the ionic contribution to the frequency dependent dielectric permittivity of clay. This model is based on a separation of timescales, and accounts for the two possible solvation modes (inner/outer-sphere complexes) for ions in the interlayer spacing, and a possible chemical exchange between both forms. The influence on the permittivity of thermodynamic (distribution constant $K_{d}$ ) and dynamic (diffusion coefficient, chemical relaxation rate) parameters is discussed. In turn, this model is used to analyze experimental data obtained with Na-Montmorillonite for two relative humidities. The values of the parameters extracted from these measurements, and their variation with water content, show that the proposed model is at least reasonable.
\end{abstract}

\section{Introduction}

Clay minerals, and particularly smectites, have received a great deal of attention in the last decades, both for their potential use for various applications -among them the in-depth storage of nuclear waste- and for their interest as a paragon for complex heterogeneous materials. They now stand for a "case-study" of water under extreme confinement conditions, although numerous questions remain to be solved.

Smectites are 2:1 layered silicates that consist of negatively charged stacks separated by galleries containing charge-compensating cations. Under humid atmosphere, the clay 
swells as a result of the ions hydration, which forces the interlayer distance to increase. As the water content increases, one, then two or more water layers can develop, depending mainly on the nature of the cation. The ions can then diffuse along the sheets. This movement is of particular interest, since it governs among others the ionic retention properties of clays, which are crucial in the waste disposal context. Diffusion can be coupled to an adsorption/desorption exchange at the clay surface, when several solvation modes exist for the cation: it has been shown that sodium ions can form either inner or outer sphere complexes with the clay surface, in proportions that vary with clay type and water content $[1,2,3]$.

On the experimental side however, ion dynamics is rather more complicated to probe than that of the water. Difficulties arise for several reasons, depending on each technique, as will be briefly discussed later, but one common feature is related to the dynamics itself: the timescale involved is too long for "fast" techniques such as neutron quasi-elastic scattering (QENS), and too short for "slow" techniques such as radioactive tracer diffusion. Indeed diffusion of an ion with a typical diffusion coefficient of $D \simeq 10^{-10} \mathrm{~m}^{2} \mathrm{~s}^{-1}$ over the distance separating it from the next cation $(L \simeq 1 \mathrm{~nm})$ takes place on the nanosecond range, and despite the short time needed for a particular ion to adsorb onto or desorb from the surface, such an event is rare so that the chemical exchange between different types of complexes is even longer than the nanosecond, too long to be observed in simulations (see section 1.3). Diffusion would be just in the higher limit of the timescales accessible with QENS (spin echo experiments), but the incoherent scattering cross section of proton is much higher than that of the cation, so that the experimental signal is almost entirely due to the water [4]. At the other extreme, tracer diffusion experiments provide information over times longer than the second, and any microscopic information is lost by a time averaging process $[5,6]$. Furthermore, during such a long time, an ion can explore not only the interlayer space, but also the various porosities that exist at different length scales. Finally, such experiments require to equilibrate the sample with reservoirs containing the tracers, and are therefore well defined only for water-saturated sample, in which pore diffusion might be the dominant mechanism.

Only nuclear magnetic resonance (NMR) on the one hand, and broadband dielectric spectroscopy (BDS) on the other hand, allow so far to explore intermediate timescales. Both techniques have their own advantages and drawbacks. Nuclear quadrupolar relaxometry (NQR) has proved very useful to trace the diffusion of a given type of ion $\left({ }^{7} \mathrm{Li}^{+}\right.$, $\left.{ }^{23} \mathrm{Na}^{+},{ }^{133} \mathrm{Cs}^{+}\right)[7,8]$, but the presence of paramagnetic impurities $\left(\mathrm{Fe}^{\mathrm{III}}\right)$ in the clay sheets disturbs the local magnetic fields [9], so that the usual sequences, involving magnetic field gradients, used for NQR cannot be employed when the cations are located in the vicinity of the clay sheets; it is therefore relevant only for studies of very hydrated (gels) samples, 
in which a majority of ions is not perturbed by the sheets. BDS has the advantage of being sensitive to all charge fluctuations, as will be explained in detail later, regardless of the charge's environment, and can therefore provide information on the ionic dynamics in the interlayer, and on structure (grain length, nature -"bound" vs. "free"- of water). However, no distinction can a priori be made between different cations bearing the same charge in a heteroionic clay; this makes its use for heteroionic clays more difficult to interpret.

The timescales accessible to BDS ranges from approximately $10 \mathrm{ps}$ to $10 \mathrm{~ms}$. The processes involved over this wide range are diverse, which makes this technique very rich, but at the same time very difficult to analyze. The assignment of each relaxation to a particular mode of charge fluctuation remains particularly challenging, unless a separation of timescales can be established. Early BDS studies of clays focused either on very high frequencies (over $10 \mathrm{GHz}$ ), where water reorientation is the dominant mechanism for charge fluctuation, or on very low frequencies $(1-10 \mathrm{kHz})$, where the "static" conductivity was investigated. The influence of clay type, cation, and water content on both properties were analyzed [10]. The intermediate frequency region, and particularly the $1 \mathrm{MHz}-$ $1 \mathrm{GHz}$, was only rarely discussed, and more thought of as a nuisance disturbing the water signal [11]. On the contrary, we argue that valuable information on the ionic dynamics could be extracted from the analysis of the dielectric spectra in this frequency range, since it corresponds precisely to the relevant timescale. More recently, investigations covering the whole frequency range where carried out, but mainly on clay suspensions [12].

The scope of the present paper is to suggest a model that would allow to extract microscopic information from the intermediate frequency region $(1 \mathrm{MHz}-1 \mathrm{GHz})$. This model accounts for the cation diffusion in the interlayer gallery and for a possible adsorption/reaction at the clay surface. Association is a critical factor governing the dynamics of the ions, since free/bound species have different mobilities. The present model is in principle not restricted to clay minerals, but could be relevant for any water containing lamellar structure, such as $\mathrm{V}_{2} \mathrm{O}_{5}$ [13], and its one-dimensional version could be applied for example to hydrated zeolites. The microscopic parameters extracted from the experimental data obtained with compacted Na-Montmorillonite samples at low water content suggest that this model is at least reasonable.

The paper is organized as follows. In section 1, the microscopic model for the interlayer is first described, and the relation between charge fluctuations (charge dynamical structure factor $\left.S_{c c}(k, \omega)\right)$ and dielectric spectroscopy is presented. In Section 2, $S_{c c}(k, \omega)$ is explicitly derived in the framework of the considered microscopic model, and the corresponding frequency dependent conductivity and permittivity are then deduced. Section 3 describes the influence of the two main parameters on the expected BDS spectra. Finally, 
the model is used in Section 4 to analyze the experimental data obtained on compacted

Na-Montmorillonite samples, and its relevance is discussed.

\section{Theoretical considerations}

\section{$1.1 \quad$ Microscopic model}

Although the question of the nature of the current carriers in the interlayer gallery has been the subject of a passionate debate [14, 10], it is now accepted that the cations rather than the protons (that would originate from cation - induced water dissociation) are responsible for electrical conduction in compacted clays [15]. We restrict ourselves to homoionic clays, where only one type of cation is present. Cations are not the only charged species, because the sheets bear themselves negative charges, but the latter don't contribute to any charge transport, since at low water content they cannot move. The situation is here completely different from the case of clay suspensions, in which both types of charges (ions and clay particles) are mobile, though with very different mobilities. We furthermore restrict our discussion to the case where no salt is added to the clay. This can be achieved by appropriate washing of the sample with deionized water, as will be explained in section 4 .

Even with only one type of cation, modeling the interlayer dynamics remains challenging. Indeed, there has been evidences that the ions can adopt two solvation modes $[1,2,3]$ : they can form with the clay surface either inner-sphere complexes (ISC) in which oxygen atoms from the sheet lattice are involved in the first coordination layer of the cation, or outer-sphere complexes (OSC) in which all oxygen atoms of the first coordination shell belong to water molecules. This may not be the case for all cations $\mathrm{Cs}^{+}$shows only ISC), but is true at least for $\mathrm{Na}^{+}$. Fully solvated ions (OSC) are able to diffuse in the interlayer gallery, while bound cations are not. These different mobilities will be shown to have an influence on the dielectric properties of the material. This behavior is similar to that observed in micellar solutions, for which some cations diffuse freely around the micelles, while other are located near the micelle surface, resulting in different mobilities, and leading to a complex dielectric response [16, 17].

The cation density profiles can be obtained by numerical simulation of the interlayer. They show that the distribution of ions depends strongly on the water content. We will adopt a two-state model for the cation distribution in the interlayer, which is consistent with the ISC/OSC distinction: a particular ion can be either stuck on the clay surface (ISC), or diffusing in the interlayer gallery (OSC), with a diffusion coefficient $D$. This microscopic model is schematically pictured in figure 1. Because a particular ion can diffuse freely in the interlayer space, and adsorb onto or desorb from the clay surface, 
with rate constants $k_{+}$(adsorption) and $k_{-}$(desorption), the two types of cations can be represented by a chemical equilibrium:

$$
C_{\text {diffuses freely }} \rightleftarrows Q_{\text {fixed }}
$$

The motion perpendicular to the sheets is assumed to be entirely described in terms of this chemical exchange. The fraction of adsorbed cations is determined by the distribution constant $K_{d}$ :

$$
K_{d}=\frac{\text { fixed }}{\text { free }}=\frac{k_{+}}{k_{-}}
$$

while the relaxation rate of the chemical equilibrium (1) is simply:

$$
k_{\chi}=\frac{1}{\tau_{\chi}}=k_{+}+k_{-}
$$

We would like to draw the reader's attention to the fact that $K_{d}$ does not strictly correspond to the usual definition, since the latter also involves cations in the mesopores, while the present model only considers cations in the interlayer. Its significance, however, remains the same, namely the ratio of bound vs. mobile ions. We are aware that the description of the exchange in terms of first order kinetic processes might not be the most relevant [18], but it is the most simple, and will therefore be used as a first stage in the modelling of the coupling between diffusion and reaction.

Within this two-state model, the first level of approximation consists in averaging over water degrees of freedom (fast motion compared to that of ions, with a contribution to the dielectric properties over the $\mathrm{GHz}$ ). Thus, the only particles explicitly described in this model are the ions. The second level of approximation is to adopt a mean-field treatment, whereby an ion evolves in the average potential created by its surrounding, which encompasses the clay stacks, the water molecules and the other ions. The structural defects, responsible for the negative charge of the sheets, are assumed to be homogeneously distributed, separated by an average distance $L$ fixed by the layer charge density $\left(\sigma_{\mathrm{S}} L^{2}=\right.$ $z$, with $z$ the charge of the cation; $z=e$ for $\mathrm{Na}^{+}$).

In order to account for the correlations induced by the (rather complex) interactions between ions, we need to evaluate the average potential to which a particular ion is submitted. The main feature of the interaction between ions is that electrostatic repulsion prevents them from getting too close to each other. The simplest approximation consists in considering an infinite well-potential, which amounts to defining a distance of closest approach $\delta$ between the ions, and neglecting the "long-ranged" correlations. This distance is of course shorter than the average distance between charges $L$. If correlations between ions are weak, $\delta \ll L$, so that the width of the well-potential is approximately $L$. A more repulsive potential would result in a shorter width, which could be extracted from 
the distribution or correlation functions obtained by simulations. This average potential corresponds in fact to a state of confinement in a two-dimensional cage of width $L$.

The dynamics of the ions in the interlayer space is therefore governed by a coupling between diffusion along the sheets and reaction with the clay surface. Before going any further in the description of this dynamics, we would like to present now how it relates to the dielectric properties of the material, by briefly recalling some results of electrodynamics and linear response theory.

\subsection{Dielectric properties}

Electrical properties of a medium can be described in terms of its frequency dependent conductivity $\sigma(\omega)$, which is a complex quantity, relating the induced electrical current to an applied electric field by $\mathbf{j}(\omega)=\sigma(\omega) \mathbf{E}(\omega)$. Usually, only the real part of the conductivity is analyzed in BDS experiments; on the contrary, both the real and imaginary parts of the permittivity $\epsilon(\omega)=\epsilon^{\prime}(\omega)-i \epsilon^{\prime \prime}(\omega)$ are generally interpreted on a so-called Argand diagram representing $\epsilon^{\prime \prime}$ as a function of $\epsilon^{\prime}$. Conductivity is linked to the electrical permittivity through the relation:

$$
\sigma(\omega)=\sigma_{\mathrm{dc}}+i \omega \epsilon_{0} \epsilon(\omega)
$$

where $\sigma_{\mathrm{dc}}$ is the static conductivity. For insulating materials such as clays, static conductivity is negligible with respect to other conduction mechanisms, which are timedependent, and arise over various timescales. In the following study of relatively high frequency conductivity, $\sigma_{\mathrm{dc}}$ can be omitted. Thus, descriptions in terms of conductivity and permittivity are equivalent. More precisely, the general framework of linear response theory relates the conductivity to the equilibrium fluctuations of the electrical current through the Kubo formula [19]:

$$
\operatorname{Re}[\sigma(\omega)]=\lim _{k \rightarrow 0} \frac{1}{V k_{B} T} \frac{1}{2} \int_{-\infty}^{\infty}\left\langle\mathbf{j}_{\mathbf{c}, \mathbf{k}}(t) \cdot \mathbf{j}_{\mathbf{c},-\mathbf{k}}(0)\right\rangle e^{i \omega t} \mathrm{~d} t
$$

where $\langle\ldots\rangle$ denotes an ensemble average, and

$$
\mathbf{j}_{\mathbf{c}, \mathbf{k}}(t)=\sum_{i} z_{i} \mathbf{v}_{i}(t) \times e^{-i \mathbf{k} \cdot \mathbf{r}_{i}(t)}
$$

is the space Fourier transform of the charge current $\left(\mathbf{r}_{i}(t)\right.$ and $\mathbf{v}_{i}(t)$ are the position and velocity of ion $i$ at time $t$ ). With the definition of the time Fourier transform used in (5), we obtain the real part of the conductivity; the imaginary part can then be computed from the Kramers-Kronig relations.

The $k \rightarrow 0$ limit, so-called "hydrodynamic limit", implies that the present derivation is also considered in the $\omega \rightarrow 0$ limit. The significance of this condition will be discussed in more detail in the next section. 
On the timescale considered, the dominant charge fluctuation mechanism is the translation of the ions in the interlayer. The dipolar (water molecules) reorientation develops on a shorter timescale, and is averaged (to zero) on the nanosecond scale. At the other extreme, translation of the negatively charged clay sheets is very long compared to that of ions, and its contribution to charge fluctuations is negligible on comparatively "short" times: if any at all, this motion should be at least as slow as the slowest observed relaxation, in the $\mathrm{kHz}$ range, attributed to grain polarization. Therefore, in the 1-1000 ns range, the charge current is only related to the ion density current; in the case of homoionic clays, we simply have:

$$
\sigma(\omega)=\lim _{k \rightarrow 0} \frac{z^{2}}{V k_{B} T} \frac{1}{2} \int_{-\infty}^{\infty}\left\langle\mathbf{j}_{\mathbf{k}}(\mathbf{t}) \cdot \mathbf{j}_{-\mathbf{k}}(0)\right\rangle e^{i \omega t} \mathrm{~d} t
$$

We now recall the classical derivation of the link between current and density fluctuations (see e.g. ref. [19]). The total ionic density $\rho(\mathbf{r}, t)$ (both fixed and mobile ions) is linked to the density current through the continuity equation:

$$
\partial_{t} \rho(\mathbf{r}, t)+\nabla \cdot \mathbf{j}(\mathbf{r}, t)=0
$$

In Fourier space, this reads:

$$
\partial_{t} \rho_{\mathbf{k}}(t)-i \mathbf{k} \cdot \mathbf{j}_{\mathbf{k}}(t)=0
$$

Thus ( $l$ is the longitudinal component):

$$
k^{2}\left\langle\mathbf{j}_{\mathbf{k} l}(t) \mathbf{j}_{-\mathbf{k} l}(0)\right\rangle=\left\langle\dot{\rho}_{\mathbf{k} l}(t) \dot{\rho}_{-\mathbf{k} l}(0)\right\rangle
$$

The r.h.s. of equation (10) is the second order time derivative of the intermediate scattering function

$$
\left\langle\dot{\rho}_{\mathbf{k}}(t) \dot{\rho}_{-\mathbf{k}}(0)\right\rangle=-\frac{\mathrm{d}^{2}}{\mathrm{~d} t^{2}}\left\langle\rho_{\mathbf{k}}(t) \rho_{-\mathbf{k}}(0)\right\rangle=-N \frac{\mathrm{d}^{2}}{\mathrm{~d} t^{2}} F(\mathbf{k}, t)
$$

$F$ is the total intermediate scattering function, which encompasses both fixed and mobile cations. It can be split in self and distinct parts:

$$
\begin{array}{r}
F_{s}(\mathbf{k}, t)=\frac{1}{N}\left\langle\sum_{i} e^{-i \mathbf{k} \cdot\left(\mathbf{r}_{i}(t)-\mathbf{r}_{i}(0)\right)}\right\rangle \\
F_{d}(\mathbf{k}, t)=\frac{1}{N}\left\langle\sum_{i} \sum_{j \neq i} e^{-i \mathbf{k} \cdot\left(\mathbf{r}_{i}(t)-\mathbf{r}_{j}(0)\right)}\right\rangle
\end{array}
$$

In the $1 \mathrm{MHz}-1 \mathrm{GHz}$ region, the density fluctuations are assumed to be related to individual ionic motion, because collective effects develop on a longer timescale, exactly as long-wavelength phonons in solids as compared to local atomic vibrations. Therefore, the distinct contribution can be neglected in the evaluation of $F(\mathbf{k}, t)$ in this frequency range. 
Valuable information on slower dynamics could be inferred from the lowest frequency part of dielectric spectra $(\omega<\mathrm{MHz})$, but we will not consider it here. At time $t=0$, we simply have $F_{s}(\mathbf{k}, 0)=1$. Equation (10) combined with (11) reads in Fourier space:

$$
\left\langle\mathbf{j}_{\mathbf{k}} \mathbf{j}_{-\mathbf{k}}\right\rangle(\omega)=\omega^{2} \frac{N S(\mathbf{k}, \omega)}{k^{2}}
$$

with $S(\mathbf{k}, \omega)$ the dynamical structure factor, time Fourier transform of $F(\mathbf{k}, t)$. The real conductivity $\left(\sigma=\sigma^{\prime}+i \sigma^{\prime \prime}\right)$ can finally be cast in the form:

$$
\sigma^{\prime}(\omega)=\frac{z^{2} N}{V k_{B} T} \lim _{k \rightarrow 0} \omega^{2} \frac{S(\mathbf{k}, \omega)}{k^{2}}
$$

\subsection{Timescale separation - hydrodynamic approach}

We would like to draw the reader's attention to the following point: there is no inconsistency in using the hydrodynamic limit $(k, \omega \rightarrow 0)$ for a motion which was said to be "fast" (local vs. collective movements).

On the one hand, the low frequency limit defining the hydrodynamic approach considers times which are longer than:

1. the characteristic time of collisions between ions and the solvent, i.e. the "inertial" time for the ions velocity relaxation due to momentum transfer with the solvent; this allows a description of the ionic movements in terms of simple diffusion. Reorientation of solvent molecules are also ruled out of this description because they are too fast to be observed at this timescale.

2. the characteristic time of the elementary act of the chemical reaction, namely the motion of a particular ion perpendicularly to the surface, from the middle to the edge of the gallery (or the way back); this justifies a description of the chemical reaction in terms of a stochastic process, with well-defined rate constants.

The former is of order of picoseconds (as well as solvent reorientation), the latter is more difficult to estimate, but is shorter than the nanosecond (probably even shorter), because the interlayer spacing is very small. This doesn't imply anything for $k_{\chi}$, because a fast elementary act can be rare. This is what makes such an exchange difficult to observe

in molecular dynamics simulations, since the duration of a simulation run (hundreds of picoseconds) does not garantuee that such an event will occur during the run.

On the other hand, the considered motion is "fast" compared to other processes leading to charge fluctuations: the above-mentioned collective motions, which manifest themselves on length-scales larger than the average inter-ionic distance, or the grain polarization, need more time to develop, and the local motions considered here are thus comparatively "fast", and correspond to higher frequencies. 
There is therefore no contradiction to use the hydrodynamic approach for processes that are fast compared to others, as soon as they are slow enough to meet the criterion defining the framework of this approach. We will need to verify that the characteristic times extracted with this model from the experiments indeed satisfy this assumption. Anticipating the results, we can say that the deduced diffusion times are in the nanosecond range (far beyond picoseconds), and the deduced chemical relaxation time even longer, which justifies this treatment.

\section{Conductivity - Permittivity}

In this section, we derive explicitly $S(\mathbf{k}, \omega)$ for this reaction/diffusion model, in the considered frequency range, and then compute the corresponding conductivity and permittivity.

\subsection{Dynamic structure factor $S(k, \omega)$}

Within our mean-field treatment, the evolution of the system is governed by the following coupled equations:

$$
\begin{aligned}
\partial_{t} C & =D \nabla^{2} C-k_{+} C+k_{-} Q \\
\partial_{t} Q & =k_{+} C-k_{-} Q
\end{aligned}
$$

These equations are simply the continuity equations for mobile $(C)$ and fixed $(Q)$ species, incorporating both diffusion flux (for mobile $C$ only) and chemical reaction. This system is solved with appropriate boundary conditions, with the help of an analogy between the present system and one obeying the same evolution equations, but for a particle confined in a box $[20,21]$. Without loss of generality we now focus on the one-dimensional case; the generalization to two-dimensional confinement is trivial and will be given at the end of this section.

The boundary conditions suggest to expand the two functions $C(x, t)$ and $Q(x, t)$ between $x=0$ and $x=L$ on a basis of periodic functions with zero derivative in $x=0, L$ (no concentration gradient).

$$
\begin{gathered}
C(x, t)=C_{0}+\sum_{n=1}^{\infty} C_{n} \cos \frac{n \pi x}{L} \\
C_{0}=\frac{1}{L} \int_{0}^{L} C(x, t) \mathrm{d} x \quad ; \quad C_{n}=\frac{2}{L} \int_{0}^{L} C(x, t) \cos \frac{n \pi x}{L} \mathrm{~d} x
\end{gathered}
$$

To obtain $F(k, t)$, we first compute $C_{n}(t)$ and $Q_{n}(t)$ using equations (16) and (17), together 
with the initial conditions:

$$
\begin{aligned}
& C(x, t=0)=\frac{C_{\mathrm{tot}}}{1+K_{d}} \delta\left(x-x_{0}\right) \\
& Q(x, t=0)=\frac{C_{\mathrm{tot}} K_{d}}{1+K_{d}} \delta\left(x-x_{0}\right)
\end{aligned}
$$

Then we average over the initial conditions (i.e. all $x_{0}$ between 0 and $L$ ). Each harmonic $(n>1)$ verifies:

$$
\begin{aligned}
\frac{\mathrm{d} C_{n}}{\mathrm{~d} t} & =-\left[k_{\mathrm{diff}, n}+k_{+}\right] C_{n}+k_{-} Q_{n} \\
\frac{\mathrm{d} Q_{n}}{\mathrm{~d} t} & =k_{+} C_{n}-k_{-} Q_{n}
\end{aligned}
$$

where the following diffusion rate constant was introduced:

$$
k_{\text {diff, } n}=\frac{1}{\tau_{\text {diff, }, n}}=D\left(\frac{n \pi}{L}\right)^{2}
$$

The solution of the linear system (22-23) reads:

$$
F(k, t)=A_{0}(k L)+\sum_{n=1}^{\infty}\left(\alpha_{n, 1} e^{\lambda_{n, 1} t}+\alpha_{n, 2} e^{\lambda_{n, 2} t}\right) A_{n}(k L)
$$

with

$$
\left\{\begin{array}{l}
\alpha_{n, 1}=\frac{\left(k_{\text {diff, } n}-k_{\chi}+\sqrt{\Delta}\right)\left(k_{\text {diff, } n}+k_{\chi}-\sqrt{\Delta}\right)}{4 k_{\chi} \sqrt{\Delta}} \\
\alpha_{n, 2}=\frac{\left(k_{\text {diff, } n}+k_{\chi}+\sqrt{\Delta}\right)\left(-k_{\text {diff }, n}+k_{\chi}+\sqrt{\Delta}\right)}{4 k_{\chi} \sqrt{\Delta}} \\
\lambda_{n, 1}=-\frac{1}{\tau_{n, 1}}=-\frac{1}{2}\left(k_{\text {diff, } n}+k_{\chi}+\sqrt{\Delta}\right) \\
\lambda_{n, 2}=-\frac{1}{\tau_{n, 2}}=-\frac{1}{2}\left(k_{\text {diff, } n}+k_{\chi}-\sqrt{\Delta}\right) \\
\Delta=k_{\text {diff, } n}^{2}+k_{\chi}^{2}+2\left(\frac{K_{d}-1}{K_{d}+1}\right) k_{\chi} k_{\text {diff, } n}
\end{array}\right.
$$

and for the amplitudes:

$$
\begin{aligned}
& A_{0}(k L)=2 \frac{1-\cos k L}{(k L)^{2}} \\
& A_{n}(k L)=\frac{(2 k L)^{2}}{\left[(k L)^{2}-(n \pi)^{2}\right]^{2}}\left[1-(-1)^{n} \cos k L\right]
\end{aligned}
$$

One can check that the normalization condition $F(k, t=0)=1$ is satisfied (non trivial). This relies among others on the fact that $\alpha_{n, 1}+\alpha_{n, 2}=1$ for each $n$. Next, we have:

$$
S(\mathbf{k}, \omega)=A_{0}(k L) \pi \delta(\omega)+\sum_{n=1}^{\infty} A_{n}(k L)\left[\frac{\alpha_{n, 1} \tau_{n, 1}}{1+\left(\omega \tau_{n, 1}\right)^{2}}+\frac{\alpha_{n, 2} \tau_{n, 2}}{1+\left(\omega \tau_{n, 2}\right)^{2}}\right]
$$


The first term is an elastic contribution to the structure factor, and it does not correspond to any charge transport; it should not be taken into account in deriving the frequency dependent conductivity.

Each term of the remaining series reveals two characteristic times, which are a combination of diffusion and reaction rate constants also involving the distribution constant $K_{d}$. The relative weights of each term are a function of only two parameters: the ratio of the diffusion and reaction constants $\mu=k_{\chi} / k_{\text {diff }}$, and the distribution constant $K_{d}$. A discussion of the influence of both parameters is given in Section 3. The terms of the series decrease very fast with $n$, due to the $n^{4}$ in the denominator. We keep only the $n=1$ term, and it is easy to verify numerically that the other give a negligible contribution to the sum, at least for $k \rightarrow 0$.

Generalization to a square cage in 2D along $x$ and $y$ is straightforward: the sum now runs over two indexes $m$ and $n$, each term being weighted by $A_{m}\left(k_{x} L\right) A_{n}\left(k_{y} L\right)$, and combining the constants $\alpha_{m, n, 1}\left(\alpha_{m, n, 2}\right)$ and $\tau_{m, n, 1}\left(\tau_{m, n, 2}\right)$ obtained by replacing $k_{\text {diff, } n}$ by $k_{m, n}=k_{\text {diff, } m}+k_{\text {diff, } n}=D\left(m^{2}+n^{2}\right) \pi^{2} / L^{2}$. For the above-mentioned reason, the only terms $(m, n)=(0,1)$ and $(1,0)$ can be retained.

Finally, the contribution in the third dimension (normal to the sheets) is neglected, since any motion along that direction is supposed to be encompassed in the adsorption/desorption scheme. This is justified by the small amplitude of this motion with respect to that along the sheets: the smaller the displacement, the smaller the contribution to charge fluctuations. When the interlayer distance becomes large, the contribution of ionic motion normal to the charged surfaces can be important[22], but it is not relevant here.

\subsection{Conductivity $\sigma(\omega)$}

The total dynamic charge structure factor $S(\mathbf{k}, \omega)$ is now completely specified. Let us now consider the $k \rightarrow 0$ limit in order to obtain the conductivity. The only terms of interest are $(0,1)$ and $(1,0)$ which are identical, and in the considered limit we have $A_{1}(k L) \simeq 8 / \pi^{4}(k L)^{2}$. Averaging over all possible orientations of the sheets introduces a factor of $2 / 3$, which finally gives:

$$
\sigma^{\prime}(\omega)=\frac{2}{3} \frac{8}{\pi^{4}} \frac{N z^{2}}{V k_{B} T} L^{2}\left[\frac{\alpha_{1,1} \tau_{1,1} \omega^{2}}{1+\left(\omega \tau_{1,1}\right)^{2}}+\frac{\alpha_{1,2} \tau_{1,2} \omega^{2}}{1+\left(\omega \tau_{1,2}\right)^{2}}\right]
$$

Introducing the characteristic diffusion time $\tau_{\text {diff }}=L^{2} / \pi^{2} D$, this can be written as

$$
\sigma^{\prime}(\omega)=\frac{2}{3} \frac{8}{\pi^{2}} \frac{N z^{2} D}{V k_{B} T} \tau_{\text {diff }}\left[\frac{\alpha_{1,1} \tau_{1,1} \omega^{2}}{1+\left(\omega \tau_{1,1}\right)^{2}}+\frac{\alpha_{1,2} \tau_{1,2} \omega^{2}}{1+\left(\omega \tau_{1,2}\right)^{2}}\right]
$$


where one recognizes the Nernst-Einstein expression of conductivity $\sigma_{N E}=N z^{2} D / V k_{B} T$, though it is not completely relevant here. Indeed, the Nernst-Einstein expression is valid for a solution at infinite dilution and for "infinite" time. Thus the corresponding diffusion coefficient is not necessarily the same as the "short-time" diffusion coefficient considered in this model. The $8 / \pi^{2}$ factor, close to 1 , comes from the truncation of the series; reporting the amplitudes of all terms of the series on the first one, as usually done in neutron time of flight (TOF) experiments -retaining only an elastic contribution and a unique quasi-elastic peak- one would find exactly 1 instead [4].

As already mentioned, only the real part is generally analyzed, while both the real and imaginary permittivity are interpreted. Therefore, we don't give here the imaginary part of the conductivity and now turn to the complex dielectric permittivity.

\subsection{Permittivity $\epsilon(\omega)$}

Ionic contribution to the permittivity $\epsilon=\epsilon^{\prime}-i \epsilon^{\prime \prime}$ is obtained through relation (4): taking the real part, we have

$$
\sigma^{\prime}(\omega)=\sigma_{\mathrm{dc}}+\omega \epsilon_{0} \epsilon^{\prime \prime}(\omega) \simeq \omega \epsilon_{0} \epsilon^{\prime \prime}(\omega)
$$

in the frequency range considered, since clays are insulating materials. Thus,

$$
\epsilon^{\prime \prime}(\omega)=\frac{2}{3} \frac{8}{\pi^{2}} \frac{\sigma_{N E} \tau_{\text {diff }}}{\epsilon_{0}}\left[\frac{\alpha_{1,1} \tau_{1,1} \omega}{1+\left(\omega \tau_{1,1}\right)^{2}}+\frac{\alpha_{1,2} \tau_{1,2} \omega}{1+\left(\omega \tau_{1,2}\right)^{2}}\right]
$$

The real part can be obtained using Kramers-Kronig relation. Indeed, for a complex $f(\omega)=f^{\prime}(\omega)+i f^{\prime \prime}(\omega)$ describing a linear and causal process (such as permittivity) the following relations hold:

$$
\begin{aligned}
f^{\prime}(\omega) & =\frac{1}{\pi} \int_{-\infty}^{\infty} \frac{f^{\prime \prime}\left(\omega^{\prime}\right) \mathrm{d} \omega^{\prime}}{\omega^{\prime}-\omega} \\
f^{\prime \prime}(\omega) & =-\frac{1}{\pi} \int_{-\infty}^{\infty} \frac{f^{\prime}\left(\omega^{\prime}\right) \mathrm{d} \omega^{\prime}}{\omega^{\prime}-\omega}
\end{aligned}
$$

The integrals are to be understood as principal values for the divergence at $\omega^{\prime}=\omega$. One can summarize the results as:

$$
\epsilon(\omega)=\frac{2}{3} \frac{8}{\pi^{2}} \frac{\sigma_{N E} \tau_{\text {diff }}}{\epsilon_{0}}\left[\frac{\alpha_{1,1}}{1+i \omega \tau_{1,1}}+\frac{\alpha_{1,2}}{1+i \omega \tau_{1,2}}\right]
$$

with the parameters given in (26) for $n=1$.

The total width satisfies $\Delta \epsilon=16 \sigma_{N E} \tau_{\text {diff }} / 3 \pi \epsilon_{0}$ because $\alpha_{1,1}+\alpha_{1,2}=1$. Replacing $\sigma_{N E}$ and $\tau_{\text {diff }}$ by their expressions, and using $N L / V=1 / l$ (reciprocal interlayer distance), we simply have:

$$
\Delta \epsilon=\frac{16}{3 \pi^{4}} \frac{z^{2}}{\epsilon_{0} k_{B} T} \times \frac{1}{l} \simeq \frac{40 \mathrm{~nm}}{l}
$$


$\Delta \epsilon$ is according to this model of this order of a few tens to a few hundreds ( $l$ is of the order of a few $\AA$ ), which is verified experimentally. Furthermore, the prediction that $\Delta \epsilon$ should decrease with increasing interlayer spacing (and therefore with increasing water content) will be shown to be verified experimentally in section 4 .

\section{Discussion}

In practice, one can extract from the BDS spectra the characteristic times $\left(\tau_{1,1}\right.$ and $\left.\tau_{1,2}\right)$ and the corresponding relative amplitudes $\left(\alpha_{1,1}\right.$ and $\left.\alpha_{1,2}\right)$. This makes a set of three independent parameters (because $\alpha_{1,1}+\alpha_{1,2}=1$ ). These can be inserted in equations (26), which are then solved for the three unknowns $K_{d}, k_{\chi}$ and $k_{\text {diff }}$, or equivalently $K_{d}$, $\tau_{\chi}$ and $\tau_{\text {diff }}$. This will be done from experimental data in the section 4 . In this section, we discuss the general form of the expected permittivity in the framework of the present model, and the influence of the two main parameters (the distribution constant $K_{d}$ and the rate ratio $\mu=k_{\chi} / k_{\text {diff }}$ ) on both types of diagrams which are commonly used to analyze BDS data, namely the Argand and Bode diagrams.

\subsection{Analytical results}

Permittivity is entirely specified by the two characteristic times $\tau_{1,1}=1 / \omega_{\mathrm{HF}}$ and $\tau_{1,2}=$ $1 / \omega_{\mathrm{LF}}$ and the relative amplitudes $\alpha_{1,1}=\alpha_{\mathrm{HF}}\left(\alpha_{1,2}=\alpha_{\mathrm{LF}}\right.$ is then $\left.1-\alpha_{\mathrm{HF}}\right)$, the subscripts referring to high and low frequencies respectively. All three parameters are given by equations (26), and we now discuss their dependence on $K_{d}$ and $\mu$.

The equilibrium properties are fixed by the distribution $K_{d}$ : when adsorption on the surface is weak, $K_{d} \ll 1$, whereas for strong adsorption $K_{d} \gg 1$. Now for a given $K_{d}$, the

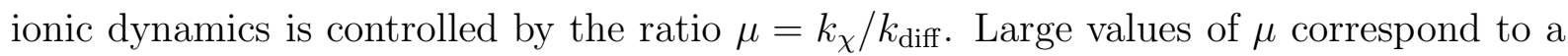
"fast" chemical exchange, i.e. a particular ion can adsorb and desorb many times before it reaches the next cation, while small $\mu$ values correspond to "slow" exchange, i.e a free ion moves back and forth many times between its neighbors before it eventually adsorbs onto the surface. Slow and fast exchange give rise to very different dielectric responses.

When the chemical exchange is slow $(\mu \ll 1)$, the two relaxations can be shown to be characterized by $\omega_{\mathrm{HF}} \simeq k_{\mathrm{diff}}$, with amplitude $\alpha_{\mathrm{HF}} \simeq 1 /\left(1+K_{d}\right)$, and $\omega_{\mathrm{LF}} \simeq k_{\chi} /\left(1+K_{d}\right)$, with amplitude $\alpha_{\mathrm{LF}} \simeq K_{d} /\left(1+K_{d}\right)$. Thus, the lower frequency (exchange in that case) depends on $K_{d}$, while the higher frequency (diffusion) doesn't. Furthermore, the contribution of each mechanism depends on the relative weights of free and fixed species. For weak adsorption $\left(K_{d} \ll 1\right)$, only the high frequency relaxation, diffusion in that case, is observed. For strong adsorption $\left(K_{d} \gg 1\right)$, only the slow process, i.e. adsorption/desorption, contributes significantly to the dielectric response. Between these two 
limiting cases, all relative amplitudes can occur.

When the chemical exchange is fast $(\mu \gg 1)$, the characteristics are now: $\omega_{\mathrm{HF}} \simeq k_{\chi}$, with amplitude $\alpha_{\mathrm{HF}} \simeq 0$, and $\omega_{\mathrm{LF}} \simeq k_{\mathrm{diff}} /\left(1+K_{d}\right)$, with amplitude $\alpha_{\mathrm{LF}} \simeq 1$. Therefore, the only observable contribution corresponds to diffusion, but with the diffusion rate (or equivalently the diffusion coefficient) weighted by the fraction of mobile cations. Fixed and mobile cations can be thought of in that case as two sides of a single entity, with an average diffusion coefficient $D_{\text {eff }}=D /\left(1+K_{d}\right)$.

These results, which constitute the main findings of the present article, are now illustrated in the two common representations used to analyze BDS data, namely the Argand and Bode diagrams.

\subsection{Argand diagram}

The Argand diagram consist in a parametric plot as a function frequency in the $\left(\epsilon^{\prime}, \epsilon^{\prime \prime}\right)$ plane. It is very convenient because a single Debye relaxation process contributing to a permittivity:

$$
\epsilon(\omega)=\frac{\Delta \epsilon}{1+i \omega \tau}
$$

appears as a semi-circle in this representation, with diameter $\Delta \epsilon$ and the frequency at the point corresponding to the maximum of imaginary part is $1 / \tau$. It is therefore straightforward to deduce both parameters with this type of diagrams. A distribution of relaxation time can also be easily extracted from this type of representation, if they are not too close to each other. The interested reader can refer to the numerous publications on the subject [23]. We only want to discuss the possibility of extracting several well-defined characteristic times.

Argand plots of the permittivity, as expected from the present calculations as a function of $\mu$ are represented in figure 2 for $K_{d}=0.1,1.0$ and 10. $\Delta \epsilon$ is independent of $K_{d}$ and $\mu$, and was fixed to unity without loss of generality. Lower frequencies are located to the right side of the figures (close to $\operatorname{Re} \epsilon=1$ ), while higher frequencies appear on the left side. Lines along the $\log \mu$ axis correspond to the same frequency.

The analytical results just presented are clearly demonstrated: for slow exchange (foreground), two distinct relaxations are observed, whose relative amplitudes depend on $K_{d}$ : for weak adsorption, the higher frequency dominates, while in the strong adsorption case, the lower frequency gives the dominant contribution; for equipartition between mobile and fixed cations, both processes contribute equally. For fast exchange (background), only one relaxation is observable. Even for slow exchange, if the distribution constant is too large $\left(K_{d} \rightarrow \infty\right)$ or too small $\left(K_{d} \rightarrow 0\right)$ only the dominant mechanism is observable, because of the $K_{d}$ dependence of the relative weights. 
To gain insight in the frequency dependence of the dielectric relaxations, we now turn to the analysis in terms of Bode diagrams.

\subsection{Bode diagram}

A Bode diagram consist in a plot of $\epsilon^{\prime \prime}$ (or $\epsilon^{\prime}$ ) as a function of frequency $(\log \omega)$. It helps to assign precisely the frequencies corresponding to each relaxation process. Bode diagrams are represented in figure 3 for $K_{d}=0.1,1$ and 10. The previous remarks on the slow and fast exchanges are also obvious from this description.

It is obvious from this representation that the dominant relaxation process depends on $K_{d}$. For increasingly fast exchange, the characteristic frequency reaches a limit, which is seen to satisfy $\omega_{\lim } \tau_{\text {diff }} \simeq 1,0.5$ and 0.1 for $K_{d}=0.1,1$ and 10 , which correspond exactly to the announced value of $1 /\left(1+K_{d}\right)$. The relaxation is in that case due to diffusion only, with a diffusion coefficient weighted by the fraction of mobile cations.

For very slow exchange, the higher characteristic frequency is $\omega_{\mathrm{HF}} \simeq k_{\text {diff, whatever }}$ the values of $K_{d}$ and $\mu$, whereas $\omega_{\mathrm{LF}}$ is proportional to $\mu$ (i.e. $\left.k_{\chi}\right)$, as can be seen from the slope of 1 in the $(\log \omega, \log \mu)$ base plane; the proportionality constant is $1 /\left(1+K_{d}\right)$, as can be inferred from the values for $\mu=10^{-2}$, namely $\omega_{\mathrm{LF}} \tau_{\text {diff }} \simeq 10^{-2}, 5.10^{-3}$ and $10^{-3}$ for $K_{d}=0.1,1$ and 10 respectively. The dominant relaxation mechanism goes from diffusion for weak adsorption $\left(K_{d} \ll 1\right)$, to exchange for strong adsorption $\left(K_{d} \gg 1\right)$; for intermediate values of the distribution constant, both mechanisms give a significant contribution.

\section{Experimental determination of $K_{d}$ and kinetic con- stants from BDS}

In order to test the validity of the present theory, we performed BDS experiments in the $40 \mathrm{~Hz}$ to $5 \mathrm{GHz}$ range on Na-Montmorillonite samples with two different water contents.

\subsection{Materials and method}

Natural Montmorillonite was obtained by purification of commercial bentonite MX-80. The size fraction $<2 \mu \mathrm{m}$ was prepared by dispersion of $40 \mathrm{~g}$ of bentonite in $1 \mathrm{~L}$ of deionized water, followed by centrifugation at appropriate velocities in order to remove mineralogical impurities and redispersed in $\mathrm{pH} 5$ water at $80^{\circ} \mathrm{C}$ under stirring for $12 \mathrm{~h}$. Na saturation of the montmorillonite suspension was carried out by dispersing the sediment in $\mathrm{NaCl} 10^{-1} \mathrm{Mol} \mathrm{L}{ }^{-1}$ solution, stirring for $12 \mathrm{~h}$ (repeated twice), and repeated washing with deionized water until complete removal of chloride anions is achieved $\left(\mathrm{AgNO}_{3}\right.$ 
tested). Since no anions are present, no cations other than that balancing the structural charge of the clay sheets can be found in the sample. The resulting sample was then dried at $80^{\circ} \mathrm{C}$ for three days and crushed. The powder is then stored under dry atmosphere. The structure of the resulting material is, as calculated by Sauzeat et al. [24], $\left(\mathrm{Si}_{7.96} \mathrm{Al}_{0.04}\right)\left(\mathrm{Al}_{3.1} \mathrm{Mg}_{0.56} \mathrm{Fe}_{0.18}^{\mathrm{III}} \mathrm{Fe}_{0.16}^{\mathrm{II}}\right) \mathrm{O}_{20}(\mathrm{OH})_{4} \mathrm{Na}_{0.76}$. The quality of purification was controlled by X-ray diffraction.

Starting with a dry sample is a crucial point, since the high complexity of the smectite hydration mechanism renders the reproducibility of the hydration step rather difficult. Indeed, the final state depends not only on the applied relative humidity $(\mathrm{RH})$, but also on the initial hydration state. Berend $[25,26]$ showed that starting from the dry state (roughly one water molecule per cation), equilibration with an atmosphere of $43 \% \mathrm{RH}$ and $85 \% \mathrm{RH}$ leads mainly to a water monolayer and bilayer respectively. Dry samples were therefore introduced for 2 weeks in a closed chamber with a given RH, obtained by equilibration over a saturated solution $\left(\mathrm{K}_{2} \mathrm{CO}_{3}\right.$ for $43 \%$ and $\mathrm{KCl}$ for $\left.85 \%\right)$. The amounts of water adsorbed by the samples were controlled by measuring the weight uptake.

The samples are then compacted in pellets with a diameter of $3 \mathrm{~mm}$ and a thickness of $1 \mathrm{~mm}$, under a pressure of 700MPa. Electrical contact with the measurement cell was achieved by the presence of a gold sheet (thickness $0.1 \mu \mathrm{m}$ ) on both faces of the pellets. The same cell was used for both low and high frequency measurements. The study of real and imaginary parts of the complex dielectric permittivity was performed in the frequency range of $40 \mathrm{~Hz}$ to $5 \mathrm{GHz}$ with two different automatic devices:

- 40Hz to 110MHz: LF impedance analyzer model Hewlett Packard HP4294A

- 45MHz to 5GHz: RF impedance analyzer model Hewlett Packard HP8510

The raw data were subsequently analyzed using a home-made program, and the deconvolution of the Argand and Bode diagrams lead to the number of relaxation and the characteristic time and amplitude of each process. As an illustration of the deconvolution process, figure 4 and 5 show the Argand diagram obtained with the Na-Montmorillonite sample equilibrated at $43 \% \mathrm{RH}$ and $85 \% \mathrm{RH}$ respectively. In $4 \mathrm{a}(5 \mathrm{a})$, the whole diagram is shown, but only the slowest relaxation (R1) is visible, because of its much larger amplitude (compared to the others, see table 1). In order to distinguish the other relaxations, one need proceed to the deconvolution of $\mathrm{R} 1$. The three faster relaxations are represented in $4 \mathrm{~b}$ (5b), together with the corresponding angular frequencies, and the deconvoluted signal corresponding to each remaining relaxations (R2, R3 and R4). Note the difference in scale between $4 \mathrm{a}(5 \mathrm{a})$ and $4 \mathrm{~b}(5 \mathrm{~b})$. Although it is not obvious from figure 4a (5a) because of the scale used here, the line is a portion of circle. The fact that some deconvoluted signals overlap (R2 and R3 in figure 4b, R3 and R4 in figure 5b) is related to the quality 
of the deconvolution. This is a source of uncertainty associated with the treatment of the signal (see section 4.2).

\subsection{Results}

For each sample (RH $43 \%$ and 85\%), four relaxations can be observed in the accessible frequency range, whose characteristics $\left(\omega_{i}\right.$ and $\left.\Delta \epsilon_{i}\right)$ are reported in table 1 . The slowest relaxation is attributed to grain polarization [15], while the fastest is attributed to water reorientation (as for bulk electrolytes), as was explained earlier. Both intermediate relaxations are assumed to correspond to the frequencies discussed in the present model. The validity of this assumption will be questioned in the next section.

The present model has been used to compute the values of the distribution constant $K_{d}$ (and the fraction of adsorbed cations $K_{d} /\left(1+K_{d}\right)$ ), the diffusion relaxation time $\tau_{\text {diff }}$ (and the diffusion coefficient D) and the chemical relaxation time. The diffusion coefficient was calculated from $\tau_{\text {diff }}$ and the average $L$ value for this clay sample, deduced from the structural charge density $\left(\sigma_{\mathrm{S}} \approx 0.262 \mathrm{Cm}^{-2}\right)$ to be $L \approx 8 \AA$.

The results reveal that the fraction of immobile cation is relatively high $(75 \%)$ for the lower RH and decreases with increasing water content, as had already been inferred from the density profiles obtained by microscopic simulations [27, 28]. Moreover, both diffusion and reaction are seen to be accelarated by the further hydration, which seems reasonable, since it is expected to facilitate all ionic movements.

A critical test is the comparison of the deduced diffusion coefficient with that obtained by molecular dynamics simulations of Na-Montmorillonite with a monolayer or a bilayer of water. The typical duration simulated in a molecular dynamics run is a little bit shorter than the nanosecond, but it remains reasonable to make a tentative comparison. The values of references $[27,28]$ are also reported in table $2\left(\mathrm{D}_{\text {sim }}\right)$. The diffusion coefficients deduced from the present model compare relatively good to that obtained by MD simulations. Although both experimental values are lower than the simulated ones, the order of magnitude is correct, and the variation with water content as well. This indicates that the present model is at least reasonable. Neutron Scattering Experiments have shown that the diffusion coefficient of water is overestimated [29] by the simulation results of references [27, 28]. This might be an indication that finding a lower value than that obtained from simulation is not entirely due to an underestimate by the present method.

As mentioned above, another possible limitation of the comparison with simulation results is the short duration of the simulation runs (typically 500ps), while the evaluated $\tau_{\text {diff }}$ are of the order of 1 ns (see table 2). In particular, there is no hope to compare the value of $\tau_{\chi}$ with simulations, because the latter cannot be deduced from simulations. Indeed, the adsorption/desorption events are to rare to be observed in simulations, and 
consequently no valuable dynamic information on the exchange process can be accessed

by simulations. Furthermore, it is difficult to determine quantitatively the fraction of adsorbed cations ( or $K_{d}$ ) from simulations, because the counter-ion profile is continuous, and the partition between free and bound cations is somewhat arbitrary. Such an issue is commonly encountered in the context of ionic association in bulk electrolytes[30]. Therefore, we restrict our comparison to the qualitative behavior of the profile upon increasing the water content, which was said to be in agreement with simulations.

It is difficult to provide a quantitative estimate of the uncertainty affecting the values given in table 2. It arises from a combination of three factors: (1) experimental uncertainty, directly related to the measurements, (2) uncertainty arising from signal processing (deconvolution), namely in assigning the values of $\alpha_{1,1}, \tau_{1,1}$ and $\tau_{1,2}$, (3) the uncertainty on these three values is transfered on the values of $K_{d}, D$ and $\tau_{\chi}$. The experimental uncertainty is expected to be small compared to that arising from the deconvolution, and is neglected. The latter is estimated to be of the order of 10 to $20 \%$ for $\tau_{1,1}$ and $\tau_{1,2}$, but much larger for the relative weights $\left(\alpha_{1,1}\right)$, of the order of $50 \%$. The fact that some deconvoluted signals overlap in figures $4 \mathrm{~b}$ and $5 \mathrm{~b}$ reflects that uncertainty. Interestingly, the transfer of these uncertainties to the values of $K_{d}, D$ and $\tau_{\chi}$ plays in our favor, because the latter are less affected by errors on $\alpha_{1,1}$ than on $\tau_{1,1}$ and $\tau_{1,2}$. This is due to the particular analytical form of equations (26). A quantitative estimate is rather difficult, but an upper bound would be of the order of $50 \%$, which means that we can be confident about the order of magnitude. As a comparison, the estimated error on the diffusion coefficient obtained by MD simulations is of the order of 10 to $15 \%$ [31].

\subsection{Discussion}

The values of $K_{d}, D$ and $\tau_{\chi}$, as extracted from the present model, as well are their evolution with water content, seem to be reasonable. Therefore, there is a hope that it provides relevant information on the microscopic dynamics of the ion in the interlayer. However, there are a number of limitations in the confidence that we can give to these results, which we now want to address.

First, as was already mentioned the present model is relevant for homoionic clays only, since a mixture of ions would introduce even more uncertainty in the assignement of the various relaxations.

Second, all the above results are related only to the interlayer contribution. In order to be able to compare the present theory to experiments, one would need to average the interlayer contribution with that of the clay sheets, which can be in this frequency region considered as frequency independent and small: it arises from the from atomic vibrations and electronic polarization, at much higher frequencies (IR and UV), and the value of the 
corresponding $\epsilon_{\infty}$ is less than a few units, as can be inferred from the limit value of $\epsilon$ for the BDS experiments. Therefore, we neglect the contribution of the sheets, and the value of the average permittivity of the sample is just that of the interlayer. This neglect might induce some deviations from the real situation.

More problematic is the contribution of cations located in the mesopores: if the latter are "empty" (i.e. are free of water), their contribution to the permittivity is that of vacuum, and is thus negligible. If they contain water (and that is the case), at least water adsorbed on their surface, the situation is not clear at all: this water solvates the cations facing the pores, and they can participate significantly to the overall charge fluctuations. A condition for this contribution to be negligible as well is that the number of such cations is much lower than that located in the interlayer galleries, which amounts to assuming that the clay stacks involve many sheets, i.e. assuming a high degree of crystallinity.

Measurements on better defined clays, such as synthetic fluorohectorites, might give useful information to address this point. First experimental investigations on such clays, which are better defined in terms of layer charge density, particle size and hydration states[32], suggest that the prediction that $\Delta \epsilon$ decreases with increasing water content (see section 2.3) is also verified in that case. This would give further credit to the present model, despite its great simplicity. Further experimental investigations will be presented elsewhere, since the purpose of the present article is more to lay out the theoretical model used to analyze the experimental data. A study with more relative humidities would also be helpful. Indeed, while for the first hydration stages the swelling of the interlayer occurs stepwise, the pore filling by water is continuous, so that a qualitative difference should be observable for the two types of cations with varying $\mathrm{RH}$.

Another point that we want to underline is that we did not take the distribution of relaxation times into account. We have deconvoluted the experimental signal assuming a discrete sum of Debye relaxations, whereas a better way to proceed would be to fit the dielectric response to a sum of so-called Cole-Cole relaxations. Briefly, it introduces an additional parameter $\alpha$ characterizing the width of the frequency dispersion around each relaxation frequency. An improvement of the model would be to account for the possibility of a non-zero width around each relaxation frequency. Two sources of a distribution of relaxation times could be found in inhomogeneities in the layer charge distribution and in inter-stratification i.e. simultaneous presence of mono- and bilayers, which are two known features of Montmorillonite clays [26].

Finally, we have interpreted the data assuming that both intermediate relaxations were due to the interlayer cations; however, we have shown that if the chemical exchange is too fast, or if the distribution constant is too extreme $\left(K_{d} \rightarrow 0\right.$ or $\left.K_{d} \rightarrow \infty\right)$, only one relaxation is visible. Therefore, our treatment assumes a priori that the exchange is not 
too fast, and that the equilibrium between fixed and mobile cations is not too displaced on either side. If only one relaxation is attributed to the cations, then the question of the assignement of the other observed one is raised.

With all these caveat in mind, we nevertheless wish to underline the innovative character of the present theory. To our knowledge, it is the first attempt to extract microscopic information on ion dynamics in the interlayer gallery of compacted clay sample from broadband dielectric spectroscopy in the $\mathrm{MHz}$ to $\mathrm{GHZ}$ range. Despite its simplicity, the model accounts for the two possible modes of solvation (inner/outer sphere complexes), and suggest a simple experimental determination of all characteristics of the chemical exchange between both forms: both thermodynamic (distribution constant $K_{d}$ ) and dynamic (chemical relaxation rate $k_{\chi}$ and diffusion coefficient $D$ ) parameters can be assigned independently in a single experiment. Not many other techniques can pretend to provide such informations, either because the relevant timescale not accessible to other spectroscopies (UV, IR, QENS), or because specific technical limitations prevent their use for compacted clays at low water content (NQR). The advantage of BDS is that it covers a large frequency range, and therefore provides information on dynamics over various time and space scales, which is necessary for multi-scale materials such as clays.

\section{Conclusion}

The ionic contribution to the complex permittivity, in the $\mathrm{MHz}-\mathrm{GHz}$ range, of compacted clay at low relative humidity, was analytically calculated from a simple two-state model, which accounts for the two possible solvation modes of the compensating cations in the clay interlayer gallery, and for the possible chemical exchange between these two forms. The local ionic dynamics was decorrelated from that (faster) of water, and that (slower) of collective ionic dynamics (giving rise to grain polarization). Based on this separation of timescale, the electric conductivity and dielectric permittivity were related to the ionic dynamic structure factor, and evaluated analytically.

The influence of the relative rates of diffusion and exchange on the one hand, and of the distribution constant $K_{d}$ between fixed and mobile cations on the other hand, on the Argand and Bode diagrams -commonly used to analyze BDS data- was discussed. For slow exchange, the dominant mechanism is diffusion for weak adsorption, and exchange for strong adsorption; for intermediate values of $K_{d}$, both mechanisms give a significant contribution. For fast exchange, the only observable relaxation is due to diffusion, with a diffusion coefficient weighted by the fraction of mobile cations.

A method was suggested to extract both thermodynamic $\left(K_{d}\right)$ and dynamic (diffusion coefficient $D$ and chemical relaxation rate $k_{\chi}$ ) informations from experimental data. This 
method was applied to experiments performed on Na-Montmorillonite at two relative

humidities, and the results suggests that despite its simplicity, and the various limitations that were discussed, the present model may provide reasonable information on the local ionic dynamics.

\section{Acknowledgments}

B.R. acknowledges financial support of the Ecole Normale Supérieure de Paris. All authors acknowledge financial support from the Groupement de Recherches PARIS. Useful discussions with Virginie Marry and Natálie Malíková are gratefully acknowledged. 


\section{References}

[1] N.T. Skipper, G. Sposito, F.C. Chang, Clays Clay Miner. 43, 294 (1995).

[2] F.C. Chang, N.T. Skipper, G. Sposito, Langmuir 11, 2734 (1995).

[3] E.S. Boek, P.V. Coveney, N.T. Skipper, J. Am. Chem. Soc. 117, 12608 (1995).

[4] M. BÉE, Quasi-elastic neutron scattering; Principles and Applications in Solid State Chemistry, Biology and Material Science, Adam Hilger, 1988.

[5] M. Molera, T. Eriksen, Radio. Acta 90, 753 (2004).

[6] I. C. Bourg, A. C. M. Bourg, G. Sposito, J. Cont. Hyd. 61, 293 (2003).

[7] P. Porion, M. P. Faugere, E. Lecolier, B. Gherardi, A. Delville, J. Phys. Chem. B 102, 3477 (1998).

[8] A. Delville, P. Porion, A.-M. Faugere, J. Phys. Chem. B 104, 1546 (2000).

[9] R. Touillaux, P. Salvador, C. Vandermeersche, J.-J. Fripiat, Israel J. Chem. 6, 337 (1968).

[10] S. D. Logsdon, D. A. Laird, Clays Clay Miner. 52, 411 (2004).

[11] G. Sposito, R. Prost, Chemical Reviews 82, 553 (1982).

[12] T. Ishida, T. Makino, C. Wang, Clays Clay Miner. 48, 7584 (2000).

[13] J.-C. Badot, A. Fourrier-Lamer, N. Baffier, J. Phys. 46, 2107 (1985).

[14] J.-J. Fripiat, A. Jelli, G.Poncelet, J. André, J. Phys. Chem. 69, 2185 (1965).

[15] P. D. Kaviratna, T. J. Pinnavaia, P. A. Schroeder, J. Phys. Chem. Solids 57, 1897 (1996).

[16] C. Baar, R. Buchner, W. Kunz, J. Phys. Chem. B 105, 2906 (2001).

[17] C. Baar, R. Buchner, W. Kunz, J. Phys. Chem. B 105, 2914 (2001).

[18] P. Levitz, J. P. Korb, D. Petit, Eur. Phys. J. E 12, 29 (2003).

[19] J. P. Hansen, I. R. McDonald, Theory of simple liquids, Academic Press, 1986.

[20] P. L. Hall, D. K. Ross, Mol. Phys. 36, 1549 (1978). 
[21] P. L. Hall, D. K. Ross, Mol. Phys. 42, 673 (1981).

[22] B. Rotenberg, J.-F. Dufrêche, P. Turq, submitted.

[23] A. K. Jonscher, Dielectric Relaxation in Solids, Chelsea Dielectrics Press, 1983.

[24] E. Sauzeat, D. Gulllaume, A. Neaman, C. Peiffert, R. Ruck, J. Dubessy, M. Cathelineau, F. Villiéras, J. Yvon, MX-80: Une référence méthodologique pour l'ANDRA, Note Technique ANDRA, 2001.

[25] I. BÉRend, Les Mécanismes d'hydratation de montmorillonites homoioniques pour des pressions relatives inférieures a 0.95, Thèse de doctorat, Institut National Polytechnique de Lorraine, 1991.

[26] J.M. Cases, I. Bérend, M. Francois, J.P. Uriot, F. Thomas, J. E. POIRIER, Langmuir 8, 2730 (1992).

[27] V. Marry, P. Turq, T. Cartailler, D. Levesque, J. Chem. Phys. 117, 3454 (2002).

[28] V. Marry,P. Turq, J. Phys. Chem. B 107, 1832 (2003).

[29] N. Malikova, A. Cadéne, V. Marry, E. Dubois, P. Turq, J.-M. Zanotti, S. Longeville, Chem. Phys., in press.

[30] A. A. Chialvo, P. T. Cummings, H. D. Cochran, J. M. Simonson, R. E. Mesmer, J. Chem. Phys. 103, 9379 (1995).

[31] N. Malikova, V. Marry, J.-F. Dufrêche, C. Simon, P. Turq, E. Giffaut, Mol. Phys. 102, 1965 (2004).

[32] J. Breu, W. Seidl, A. Stoll, Z. Anorg. Allg. Chem. 629, 503 (2003). 


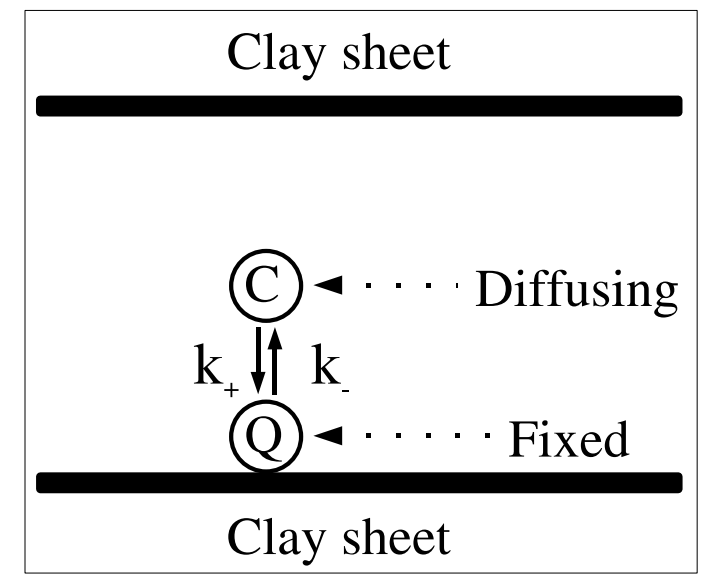

Figure 1: Schematic representation of the microscopic two-state model: in the interlayer spacing, cations can either be fixed (Q) or diffusing along the sheets (C). A particular ion exchanges between both states with rate constants $k_{+}$(adsorption) and $k_{-}$(desorption). The distribution constant between fixed and mobile cations is simply $K_{d}=k_{+} / k_{-}$.

\begin{tabular}{|c|c|c|}
\hline $\mathrm{RH}$ & $\omega_{i}\left({\mathrm{rd} . \mathrm{s}^{-1}}^{-1}\right.$ & $\Delta \epsilon_{i}$ \\
\hline \hline \multirow{4}{*}{$43 \%$} & $2.310^{3}$ & $2.910^{5}$ \\
& $2.610^{7}$ & 74 \\
& $7.010^{8}$ & 19 \\
& $3.510^{9}$ & 3 \\
\hline \multirow{4}{*}{$85 \%$} & $2.810^{4}$ & $5.010^{5}$ \\
& $1.610^{8}$ & 49 \\
& $1.510^{9}$ & 17 \\
& $1.810^{10}$ & 6 \\
\hline
\end{tabular}

Table 1: Experimental relaxation frequencies and amplitudes for Na-Montmorillonite equilibrated at $43 \%$ and $85 \%$ relative humidity.

\begin{tabular}{|l|c|c|c|c|c||c|}
\hline $\mathrm{RH}$ & $K_{d}$ & $\%$ adsorbed & $\tau_{\text {diff }}(\mathrm{ns})$ & $\tau_{\chi}(\mathrm{ns})$ & $\mathrm{D}\left(\mathrm{m}^{2} \mathrm{~s}^{-1}\right)$ & $\mathrm{D}_{\text {sim }}\left(\mathrm{m}^{2} \mathrm{~s}^{-1}\right)$ \\
\hline \hline $43 \%$ & 2.8 & 75 & 1.6 & 8.8 & $4.10^{-11}$ & $7.10^{-11}$ \\
\hline $85 \%$ & 1.4 & 60 & 0.8 & 2.1 & $8.10^{-11}$ & $6.10^{-10}$ \\
\hline
\end{tabular}

Table 2: Parameters deduced from the present model for Na-Montmorillonite equilibrated at $43 \%$ and $85 \%$ relative humidity. $D$ is obtained from $\tau_{\text {diff }}$ and the mean inter-ionic distance $L$ (see text). The diffusion coefficients obtained by simulation are reported from references [27, 28]. 
(a) $\mathrm{K}_{\mathrm{d}}=0.1$

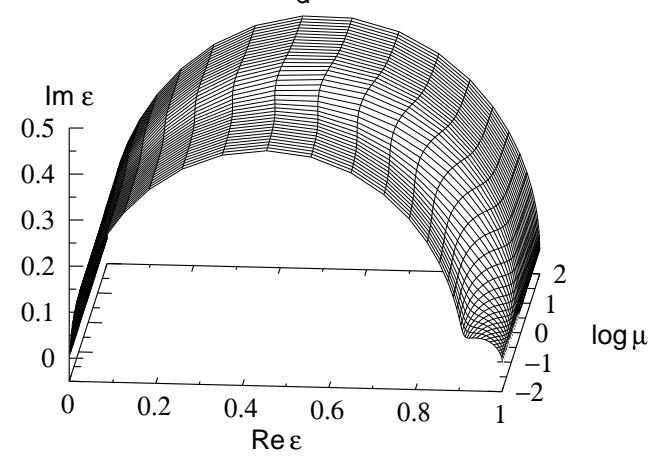

(b) $\mathrm{K}_{\mathrm{d}}=1.0$

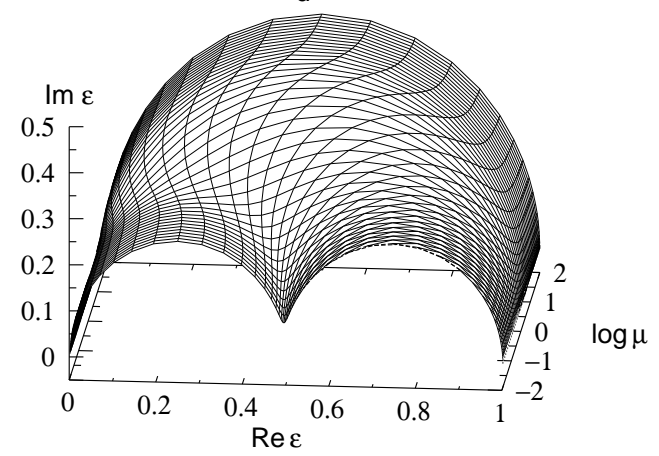

(c) $\mathrm{K}_{\mathrm{d}}=10$

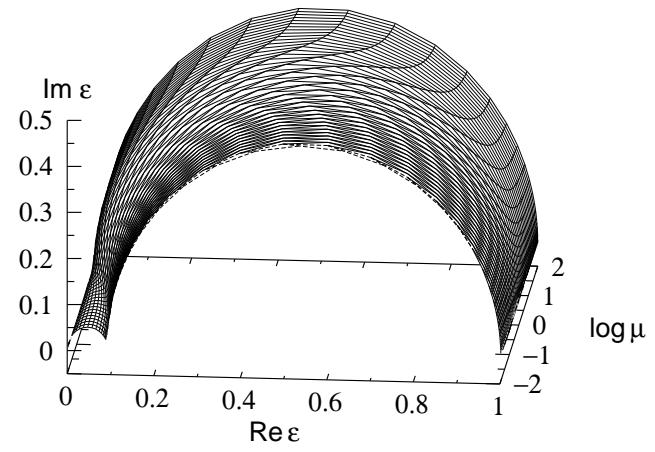

Figure 2: Argand diagrams for $K_{d}=0.1$ (a), 1.0 (b) and 10 (c). For slow exchange $(\mu \ll 1)$, two relaxations are visible, whose relative weights depend on $K_{d}$ (see text). For fast exchange $(\mu \gg 1)$, a single relaxation is observed. Lines along the $\log \mu$ axis correspond to isofrequency curves. 
(a) $\mathrm{K}_{\mathrm{d}}=0.1$

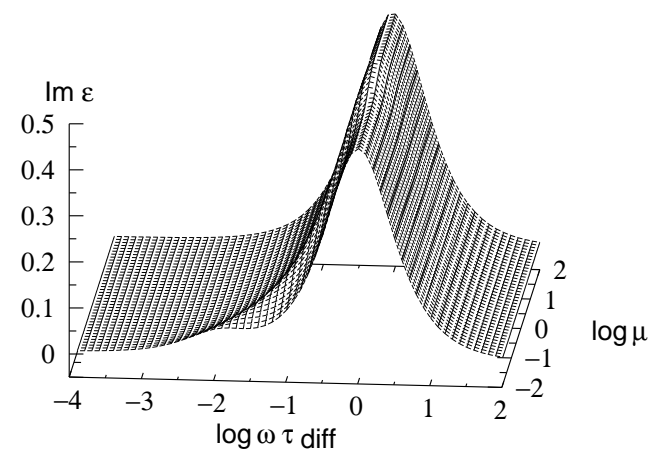

(b) $K_{d}=1.0$

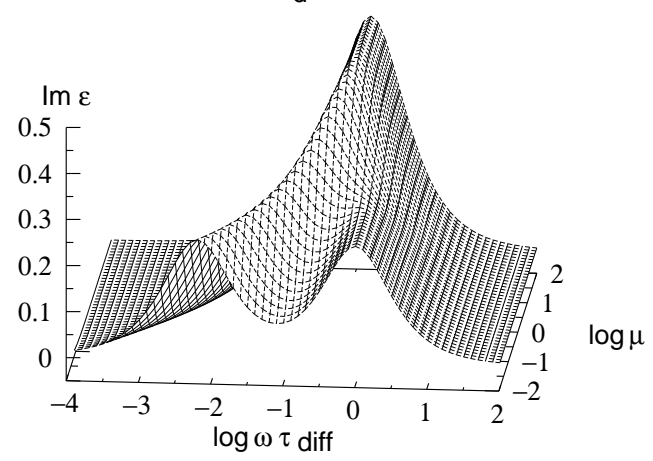

(c) $\mathrm{K}_{\mathrm{d}}=10$

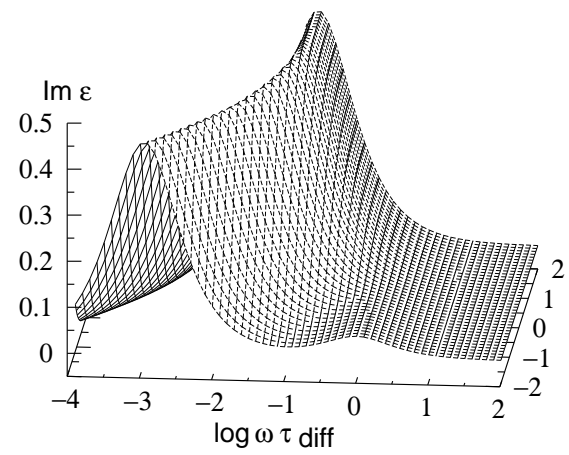

$\log \mu$

Figure 3: Bode diagrams for $K_{d}=0.1$ (a), 1.0 (b) and 10 (c). For slow exchange $(\mu \ll 1)$, the dominant mechanism is diffusion for weak adsorption (a), and exchange for strong adsorption (c); for intermediate values of the distribution constant, both mechanisms give a significant contribution (b). For fast exchange $(\mu \gg 1)$, the relaxation is due to diffusion only, with a diffusion coefficient weighted by the fraction of mobile cations (see text). 

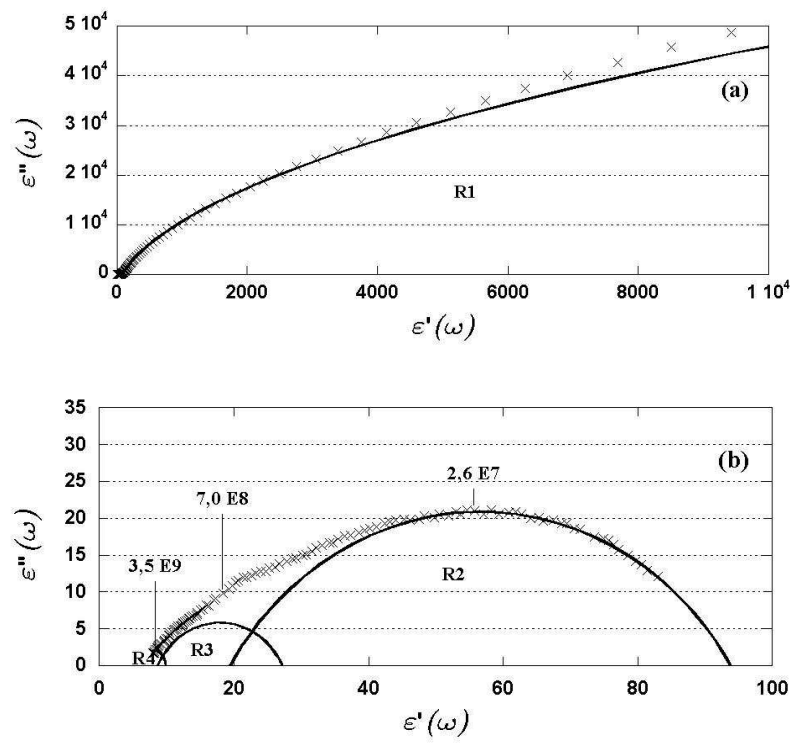

Figure 4: Experimental Argand diagram (crosses) obtained with Na-Montmorillonite at $43 \%$ relative humidity, and deconvoluted signal for the four relaxations (lines). The whole diagram is presented in (a), but only the first relaxation R1, which corresponds to lower frequencies, is visible. In (b), the experimental signal is shown after deconvolution of R1 (note the difference in scale); the contribution of the three remaining relaxations is given, together with the corresponding angular frequencies (in $\mathrm{rd} . \mathrm{s}^{-1}$ ). The parameters of the deconvolution are summarized in table 1. 

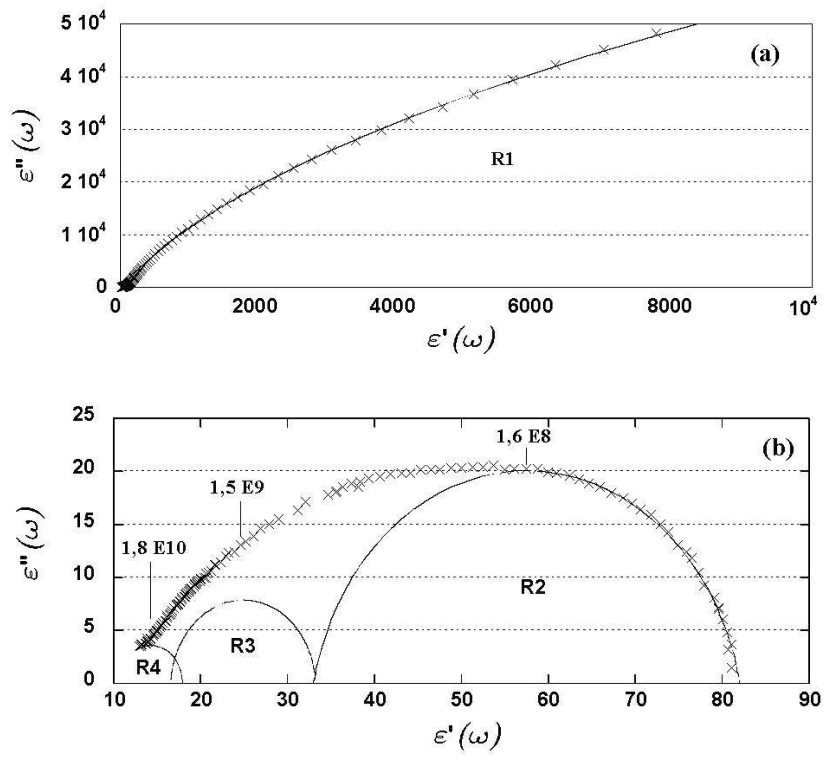

Figure 5: Same as figure 4 for Na-Montmorillonite at $85 \%$ relative humidity. The parameters of the deconvolution are summarized in table 1. 